\title{
On Polynomial Approximation in the Complex Plane with Application to Conformal Mapping
}

\author{
By Lothar Reichel
}

\begin{abstract}
We consider the selection of polynomial bases for polynomial approximation of analytic functions on bounded simply connected regions in the complex plane. While a monomial basis may be very ill-conditioned, we show that a basis of Lagrange polynomials with Fejerr points as nodes is well-conditioned. Numerical examples, where we compute polynomial approximations of conformal mappings, conclude the paper.
\end{abstract}

1. Introduction. Important for the success of any numerical method for polynomial approximation is the selection of a well-conditioned polynomial basis. For polynomial approximation on the real axis the numerical condition of various bases has been investigated by Gautschi [7], [8], [9]. We consider the numerical condition of polynomial bases for approximation on bounded simply connected regions $\Omega$ of the complex plane $\mathbf{C}$. The polynomials of a well-conditioned basis depend on the shape of $\Omega$. For disks with radius $\rho$ and center $z=0$, the monomials $p_{k}(z):=(z / \rho)^{k}$, $k=0,1,2, \ldots$ are well-conditioned, see Example 2.1 below, but on flat ellipses this basis is severely ill-conditioned, see Example 2.2. We will show that a basis of Lagrange polynomials

$$
l_{k}(z):=\prod_{\substack{j=0 \\ j \neq k}}^{n} \frac{z-z_{j}}{z_{k}-z_{j}}, \quad k=0(1) n,
$$

where the $z_{k}$ are Fejér points on the boundary of the region, is well-conditioned and simple to compute. Fejér points are defined as follows, see also Gaier [6]. Denote by $\partial \Omega$ the boundary of $\Omega$ and assume that $\partial \Omega$ is a Jordan curve. Let $\bar{\Omega}:=\Omega \cup \partial \Omega$ and $\Omega_{c}:=\mathrm{C} \backslash \bar{\Omega}$. Let $w=\psi(z)$ be a conformal map from $\Omega_{c}$ to $|w|>1$ with $\psi(\infty)=\infty$. $\psi$ can be continued continuously to a bijective map from $\Omega_{c} \cup \partial \Omega$ to $|w| \geqslant 1 . \psi$ is uniquely determined by the requirement $\psi\left(z_{1}\right)=1$. The points $\left\{z_{k}\right\}_{k=1}^{n}$ are called Fejér points if they satisfy

$$
\psi\left(z_{k}\right)=\exp (2 \pi i(k-1) / n), \quad k=1(1) n .
$$

Note that $z_{1}$ is an arbitrary point on $\partial \Omega$. To determine a set of Fejér points, we need the restriction of $\psi$ to $\partial \Omega$. This restriction we compute by solving a modified Symm's

Received January 23, 1984; revised May 23, 1984.

1980 Mathematics Subject Classification. Primary 41A10, 65D05, 30C30.

Key words and phrases. Polynomial approximation, polynomial basis, numerical condition, conformal mapping. 
integral equation. The modification makes Symm's integral equation uniquely solvable.

We next turn to our application, the polynomial approximation of conformal mappings from $\Omega$ to the unit disk. Besides illustrating the good numerical condition of the Lagrange basis (1.1), polynomial approximants of conformal mappings are important in themselves. They provide an approximation of particularly simple form, easy to evaluate at interior as well as at boundary points. Assume from now on that $\Omega$ is open and contains the origin. Let $w=\phi(z)$ denote a conformal mapping from $\Omega$ to $|w|<1$, such that $\phi(0)=0$. $\phi$ can be continued continuously to a bijective map from $\bar{\Omega}$ to $|w| \leqslant 1$. The restriction of $\phi$ to $\partial \Omega$ we compute by a modified Symm's integral equation and determine a polynomial approximant of $\phi$ by interpolation in a set of Fejér points. The norm of this interpolation projection grows only logarithmically with the degree of the polynomial, see Theorem 2.1. The numerical scheme we present to compute the polynomial approximant requires only little computation once a set of Fejér points has been determined.

The paper is organized as follows. Section 2 contains results on the numerical condition of some polynomial bases. In Section 3, we introduce the modified Symm's integral equation, and in Section 4, we present the numerical scheme for computing polynomial approximants to conformal mappings. This section also contains computed examples.

2. Condition of Some Polynomial Bases. We consider the numerical condition of the Lagrange basis with Fejér nodes, the monomial basis and a basis of Newton polynomials with Fejér nodes. Let $q_{j}(z), j=0(1) n$ be polynomials such that $\operatorname{span}\left\{q_{j}\right\}_{j=0}^{n}=\operatorname{span}\left\{z^{j}\right\}_{j=0}^{n}$. We wish to investigate the sensitivity of

$$
Q_{n}(z):=\sum_{j=0}^{n} a_{j} q_{j}(z), \quad z \in \bar{\Omega},
$$

with respect to perturbations in the coefficients $a_{j}$. Following Gautschi [9], we introduce the map $M_{n}: \mathbf{C}^{n+1} \rightarrow \Pi_{n}$, mapping the coefficient space on the space of polynomials of degree $\leqslant n$. With $\mathbf{a}:=\left(a_{0}, a_{1}, \ldots, a_{n}\right) \in \mathbf{C}^{n+1}$, we define

$$
\left(M_{n} \mathbf{a}\right)(z):=\sum_{k=0}^{n} a_{k} q_{k}(z), \quad z \in \bar{\Omega} \text {. }
$$

For the inverse of $M_{n}$, we have $M_{n}^{-1} Q_{n}=\mathbf{a}$. In $\mathbf{C}^{n+1}$ we introduce the maximum norm $\|\mathbf{a}\|_{\infty}:=\max _{0 \leqslant k \leqslant n}\left|a_{k}\right|$. Also $\Pi_{n}$ we equip with the maximum norm $\left\|Q_{n}\right\|_{\partial \Omega}:=\sup _{z \in \partial \Omega}\left|Q_{n}(z)\right|$. Let $\left\|M_{n}\right\|$ and $\left\|M_{n}^{-1}\right\|$ be the induced operator norms. Our interest of this section is how the condition of the map $M_{n}$, defined by

$$
\text { cond } M_{n}:=\left\|M_{n}\right\|\left\|M_{n}^{-1}\right\| \text {, }
$$

grows with $n$ for various choices of $q_{j}$.

THEOREM 2.1. Let $q_{k}(z)$ be the Lagrange polynomials (1.1) with the $z_{k}$ being Fejerr points, and assume that $\partial \Omega$ is analytic. Then, for all $n \geqslant 1$,

$$
\text { cond } M_{n} \leqslant \frac{2}{\pi} \ln n+\alpha
$$

where $\alpha$ is a constant depending on the shape of $\Omega$. 
Proof. The theorem may be regarded as a corollary to Theorem B of Curtiss [2]. We first note that $\left\|M_{n}^{-1}\right\|=1$ and therefore

$$
\text { cond } M_{n}=\left\|M_{n}\right\|=\left\|\sum_{k=0}^{n}\left|l_{k}(z)\right|\right\|_{\partial \Omega} .
$$

Curtiss [2] shows that when $f$ is a function analytic in $\Omega$, continuous on $\bar{\Omega}$ and of bounded variation on $\partial \Omega$, then the interpolation projection

$$
\left(L_{n} f\right)(z):=\sum_{k=0}^{n} f\left(z_{k}\right) l_{k}(z)
$$

converges to $f(z)$ in norm $\|\cdot\|_{\partial \Omega}$. Combining (3.3), (3.6), (4.2) and (4.6) of Curtiss [2], one obtains after some simplifications

$$
\left\|L_{n}\right\| \leqslant\left\|L_{n}^{d}\right\|+\beta+O\left(r^{n}\right), \quad n \rightarrow \infty,
$$

where $0<r<1, \beta \geqslant 0$ are constants depending on the location of the point set in $\Omega$ on which no analytic continuation of the conformal map $\psi$ can be defined. $L_{n}^{d}$ denotes the interpolation projection for the case when $\Omega$ is the unit disk. The theorem now follows from $\left\|L_{n}^{d}\right\| \leqslant(2 / \pi) \ln n+0.08+o(1)$; see Geddes and Mason [11].

Remark 2.1. $\left\|L_{n}\right\|$ has been computed numerically for ellipses (Geddes [10]), for polygons (Afolabi and Geddes [1]), and for a semidisk (Elliott [4]). Also for these latter contours with corners $\left\|L_{n}\right\|$ was found to grow like $\ln n, n \rightarrow \infty$. A survey of these and related results has recently been given by Mason [14].

We next turn to the monomial basis

$$
q_{j}(z):=c_{j} z^{j}, \quad j=0,1,2, \ldots,
$$

where we choose real positive coefficients $c_{j}$ so that $\left\|q_{j}\right\|_{\partial \Omega}=1$.

Example 2.1. Let $\Omega$ be the unit disk. Then

$$
\begin{gathered}
\left\|M_{n}\right\|=\max _{\|\mathbf{a}\|_{\infty}=1}\left\|\sum_{j=0}^{n} a_{j} z^{j}\right\|_{\partial \Omega}=n+1, \\
\left\|M_{n}^{-1}\right\|=\left(\min _{\|\mathbf{a}\|_{\infty}=1}\left\|\sum_{j=0}^{n} a_{j} z^{j}\right\|_{\partial \Omega}\right)^{-1} \geqslant 1 .
\end{gathered}
$$

From

$$
\left\|Q_{n}\right\|_{\infty} \geqslant\left(\frac{1}{2 \pi} \int_{0}^{2 \pi}\left|Q_{n}\left(e^{i \theta}\right)\right|^{2} d \theta\right)^{1 / 2}=\left(\sum_{j=0}^{n}\left|a_{j}\right|^{2}\right)^{1 / 2}
$$

we obtain

$$
\left\|M_{n}^{-1}\right\| \leqslant\left(\min _{\|\mathbf{a}\|_{\infty}=1}\left(\sum_{j=0}^{n}\left|a_{j}\right|^{2}\right)^{1 / 2}\right)^{-1}=1 .
$$

Hence, cond $M=n+1$, showing that the basis is fairly well-conditioned.

Example 2.2. Let $\partial \Omega$ be the ellipse $E(c, d):=\left\{z=x+i y,(x / c)^{2}+(y / d)^{2}=1\right\}$, where $c, d$ are real constants, $c \geqslant d$. The scaling factors are $c_{k}:=c^{-k}$ which is the reason for introducing the new variable $w:=z / c$. Then $w \in E(1, d / c)$, an ellipse 
with foci $\pm f$, where $f=\sqrt{1-(d / c)^{2}}$. The Chebyshev polynomials for $E(1, d / c)$ with highest-order coefficient 1 are

$$
\begin{array}{r}
T_{n}(w, f):=\frac{f^{n}}{2^{n}-1} T_{n}\left(\frac{w}{f}\right)=\frac{1}{2^{n}}\left(\left(w+\sqrt{w^{2}-f^{2}}\right)^{n}+\left(w-\sqrt{w^{2}-f^{2}}\right)^{n}\right) \\
n=0,1,2, \ldots
\end{array}
$$

where $T_{n}(\cdot)$ is the Chebyshev polynomial of the first kind. Write the points $w \in E(1, d / c)$ as

$$
w=\frac{1}{2}\left(\left(1+\frac{d}{c}\right) e^{i \theta}+\left(1-\frac{d}{c}\right) e^{-i \theta}\right), \quad 0 \leqslant \theta<2 \pi,
$$

and substitute into (2.8). This yields

$$
T_{n}(w, f)=\left(\frac{1}{2}\left(1+\frac{d}{c}\right)\right)^{n} e^{i n \theta}+\left(\frac{1}{2}\left(1-\frac{d}{c}\right)\right)^{n} e^{-i n \theta} \sim\left(\frac{1}{2}\left(1+\frac{d}{c}\right)\right)^{n} e^{i n \theta},
$$

and therefore,

$$
\left\|T_{n}(w, f)\right\|_{E(1, d / c)} \sim\left(\frac{1}{2}\left(1+\frac{d}{c}\right)\right)^{n}, \quad n \rightarrow \infty .
$$

Let $\tau^{(n)}:=\left(\tau_{0}^{(n)}, \tau_{1}^{(n)}, \ldots, \tau_{[n / 2]}^{(n)}\right)$ denote the coefficient vector of $T_{n}(w, f)$,

$$
T_{n}(w, f)=\sum_{k=0}^{[n / 2]} \tau_{k}^{(n)} w^{n-2 k}, \quad \text { where }[s] \text { denotes the largest integer } \leqslant s .
$$

For $f=0$, one has $\tau_{0}^{(n)}=1$, and $\tau_{k}^{(n)}=0, k>0$. This corresponds to the ellipse being a circle, a case that has been treated in the previous example. Assume $f>0$. A lower bound for $\left\|M_{n}^{-1}\right\|$ is obtained from

$$
\left\|M_{n}^{-1}\right\|=\left(\min _{\|\mathbf{a}\|_{\infty}=1}\left\|\sum a_{k} w^{k}\right\|_{E(1, d / c)}\right)^{-1} \geqslant \frac{\left\|\boldsymbol{\tau}^{(n)}\right\|_{\infty}}{\left\|T_{n}\right\|_{E(1, d / c)}} .
$$

Previously Gautschi [9, Eq. (2.2)] has used this bound to obtain estimates for the numerical condition of the monomial basis on intervals $[-c, c]$. We use the estimate for $\left\|\tau^{(n)}\right\|_{\infty}$ derived in [9],

$$
\left\|\tau^{(n)}\right\| \sim \sqrt{\frac{2}{\pi}} \cdot \frac{\left(1+f^{2}\right)^{3 / 4}}{f} n^{-1 / 2}\left(\frac{1+\sqrt{1+f^{2}}}{2}\right)^{n}, \quad n \rightarrow \infty .
$$

Combining (2.9-11) and noting that $\left\|M_{n}\right\|=n+1$ yields

$$
\left\|M_{n}^{-1}\right\| \gtrsim \sqrt{\frac{2}{\pi}} \frac{\left(1+f^{2}\right)^{3 / 4}}{f} n^{-1 / 2}\left(\frac{1+\sqrt{1+f^{2}}}{1+d / c}\right)^{n}, \quad n \rightarrow \infty,
$$

and

$$
\text { cond } M_{n} \geq \sqrt{\frac{2}{\pi}} \frac{\left(1+f^{2}\right)^{3 / 4}}{f} \sqrt{n}\left(\frac{1+\sqrt{1+f^{2}}}{1+d / c}\right)^{n}, \quad n \rightarrow \infty,
$$

where $\gtrsim$ stands for asymptotically larger than or equal to. Introduce $\gamma:=d / c$ and $F(\gamma)$,

$$
\frac{1+\sqrt{1+f^{2}}}{1+d / c}=\frac{1+\sqrt{2-\gamma^{2}}}{1+\gamma}=: F(\gamma)
$$


We find that

$$
\begin{aligned}
& F(0)=1+\sqrt{2}, \quad \text { this corresponds to } \partial \Omega \text { being the interval }[-c, c] . \\
& F(1)=1 \\
& F^{\prime}(\gamma)<0, \quad 0 \leqslant \gamma \leqslant 1 .
\end{aligned}
$$

Hence, if the ellipse has not degenerated into a circle, the condition number grows exponentially with $n$, and the growth rate increases with the ratio $c / d$.

Examples 2.1 and 2.2 demonstrate that when using the monomial basis for approximation on regions not very disk-like, the ill-conditioning of the basis may cause numerical difficulties.

3. A Modified Symm's Equation. Symm's integral equation and our modification of it differ in that

(1) the modified equation is uniquely solvable for all scalings of the contour;

(2) the modified integral equations for $\left.\psi\right|_{\partial \Omega}$ ( = the restriction of $\psi$ to $\partial \Omega$ ) and for $\left.\phi\right|_{\partial \Omega}$ differ only in their right-hand sides. Therefore both restrictions can be computed to practically the cost of one. We need $\left.\psi\right|_{\partial \Omega}$ in order to allocate a set of Fejér points. $\left.\phi\right|_{\partial \Omega}$ is needed when determining polynomial approximants to $\phi$.

THEOREM 3.1. Let $\theta(z):=\arg (\psi(z))$ for $z \in \partial \Omega$, and let $\gamma$ denote the capacity of $\partial \Omega$. Then the unique solution $\left\{q^{*}, \sigma^{*}\right\}$, where $\sigma^{*}=\sigma^{*}(z) \in L^{2}(\partial \Omega)$ and $q^{*}$ is a constant, of the modified Symm's equation

$$
\left\{\begin{array}{l}
q+\int_{\partial \Omega} \ln |z-\zeta| \sigma(\zeta)|d \zeta|=0, \quad z \in \partial \Omega \\
\int_{\partial \Omega} \sigma(\zeta)|d \zeta|=1
\end{array}\right.
$$

satisfies

$$
\theta(z)=2 \pi \int_{z_{1}}^{z} \sigma^{*}(\zeta)|d \zeta|, \quad q^{*}=-\ln \gamma
$$

Integration in (3.2) is along $\partial \Omega$ in the positive direction.

Proof. Introduce the parametric representation $s \rightarrow \zeta(s)$ of $\partial \Omega, 0 \leqslant s<l, \zeta(0)=$ $z_{1}$, where $s$ is the arclength of $\partial \Omega$. For $\zeta \in \partial \Omega$ we may regard $\theta(\zeta)$ also as a function of $s, \tilde{\theta}(s):=\theta(\zeta(s))$. Gaier [5] shows that for rectifiable contours $\partial \Omega$, and $\gamma \neq 1$, the integral equation

$$
\int_{\partial \Omega} \ln |z-\zeta| v(\zeta)|d \zeta|=1 \quad \text { a.e. }
$$

has the unique, integrable solution

$$
v(\zeta)=\frac{1}{2 \pi} \cdot \frac{1}{\ln \gamma} \tilde{\boldsymbol{\theta}}^{\prime}(s), \quad \zeta=\zeta(s),
$$

where differentiation of $\tilde{\theta}$ is with respect to $s$. Therefore (3.1) has the unique solution

$$
\left\{\begin{array}{l}
\sigma^{*}(\zeta)=\frac{1}{2 \pi} \tilde{\theta}^{\prime}(s), \quad \zeta=\zeta(s), \\
q^{*}=-\ln \gamma
\end{array}\right.
$$


In [15] we showed that (3.1) has a unique solution for any scaling of $\partial \Omega$, and that $\sigma^{*}$ is invariant under scaling and $q^{*}$ varies continuously with the scaling. Therefore (3.4) is the unique solution also for $\gamma=1$.

TheOREM 3.2. Let $\theta_{i}(z):=\arg (\phi(z))$ for $z \in \partial \Omega$ with $\theta_{i}\left(z_{1}\right)=0$. Then the unique solution $\left\{q_{i}^{*}, \sigma_{i}^{*}\right\}$, where $q_{i}^{*}$ is a constant and $\sigma_{i}^{*}=\sigma_{i}^{*}(z) \in L^{2}(\partial \Omega)$, of the system of integral equations

$$
\left\{\begin{array}{l}
q_{i}+\int_{\partial \Omega} \ln |z-\zeta| \sigma_{i}(\zeta)|d \zeta|=\ln |z|, \quad z \in \partial \Omega \\
\int_{\partial \Omega} \sigma_{i}(\zeta)|d \zeta|=1
\end{array}\right.
$$

satisfies

$$
\left\{\begin{array}{l}
\theta_{i}(z)=2 \pi \int_{z_{1}}^{z} \sigma_{i}^{*}(\zeta)|d \zeta| \\
q_{i}^{*}=0
\end{array}\right.
$$

Integration is along $\partial \Omega$ in the positive direction.

Proof. As in the proof of Theorem 3.1, we use results of Gaier [5]. Let $\gamma$ be the capacity of $\partial \Omega$ and assume that $\gamma \neq 1$. By Gaier [5],

$$
\int_{\partial \Omega} \ln |z-\zeta| \rho(\zeta)|d \zeta|=\ln |z|, \quad z \in \Gamma
$$

has a unique solution $\rho^{*}(\zeta)$ which, moreover, satisfies

$$
\int_{\partial \Omega} \rho^{*}(\zeta)|d \zeta|=1
$$

Therefore $\sigma_{i}^{*}=\rho^{*}$ and $q^{*}=0$ is a solution of (3.5). By [15], (3.5) has a unique solution, which is invariant under scaling. Therefore (3.6) also holds for curves with $\gamma=1$.

In [16] we describe a fast Fourier-Galerkin method for the numerical solution of (3.1) and (3.5).

4. Polynomial Approximation of the Mapping $\phi$. The numerical scheme is as follows:

1. Compute $\theta(z)$ and $\theta_{i}(z)$ defined in Theorems 3.1 and 3.2.

2. Determine $n+1$ Fejér points $z_{k}$ by solving the equations

$$
\theta\left(z_{k}\right)=2 \pi \frac{k}{n+1}, \quad k=0(1) n
$$

3. Compute the images $w_{k}$ of $z_{k}$ under $\phi$ by evaluating

$$
w_{k}:=\exp \left(i \theta_{i}\left(z_{k}\right)\right), \quad k=0(1) n \text {. }
$$

Determine a polynomial approximant $p_{n}(z)$ of $\phi(z) / z$ of degree $\leqslant n$ by interpolating $\phi(z) / z$ at the Fejér points $z_{k}, k=0(1) n$. An approximation of $\phi(z)$ is given by

$$
\phi_{n+1}(z):=z p_{n}(z)
$$


The accuracy of $\phi_{n+1}$ depends on the accuracy of $\theta_{i}(z), \theta(z)$, and on the interpolation error. In the examples, the approximation error in $\phi_{n+1}$ is generally determined by how accurately we have computed $\theta_{i}(z)$. The basis of Lagrange polynomials has not caused any problems due to ill-conditioning. We had no difficulty with polynomials of degree 80-100, despite the fact that all computations were carried out on a DEC-10 computer in single precision, i.e., with only 8 significant digits. For polynomials of such high degree the interpolation error was much smaller than the error in the computed $\theta_{i}(z)$. In the examples, we have computed $\theta_{i}(z)$ and chosen the degree of the polynomial so that the interpolation error and the error in $\theta_{i}$ are of the same order of magnitude.

When using a monomial basis, ill-conditioning caused difficulties. The monomial basis used is $\left\{(z / c)^{k}\right\}_{k=0}^{n}$, where $c:=\max _{\partial \Omega}|z|$. The condition number of the interpolation matrix

$$
A_{n}:=\left[a_{j k}\right], \quad a_{j k}=\left(z_{k} / c\right)^{j}, \quad 0 \leqslant j, k \leqslant n,
$$

is measured in the maximum norm.

Example 4.1. Let $\partial \Omega=\left\{z=2 \cos (t)+i\left(\sin (t)+2 \cos ^{3}(t)\right), 0 \leqslant t<2 \pi\right\}$.

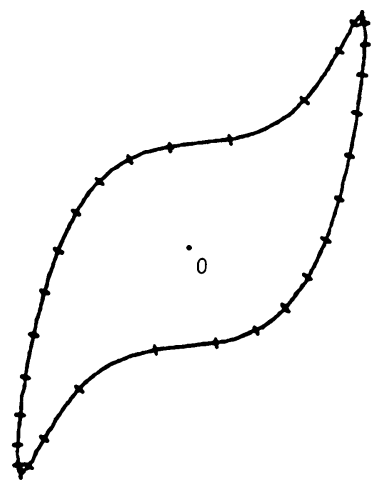

FIGURE 4.1

Figure 4.1 shows $\partial \Omega$ and 32 Fejér points marked with dashes.

$\begin{array}{ccc}n & \text { Basis* }^{*} & \left\|\left|\phi_{n}(z)\right|-1\right\|_{\partial \Omega} \\ 16 & \mathrm{M} & 3 \cdot 10^{-2} \\ 32 & \mathrm{M} & 3 \cdot 10^{-3} \\ 64 & \mathrm{~L} & 9 \cdot 10^{-6}\end{array}$

${ }^{*} \mathrm{M}=$ monomial basis, $\mathrm{L}=$ Lagrange basis.

The condition number of $A_{32}$ was $5 \cdot 10^{9}$. To obtain a significantly smaller error, the use of a better-conditioned basis is necessary.

Approximations of $\phi(z)$ using polynomials have previously been computed by Ellacott [3], who determined polynomial approximants to $\ln (\phi(z) / z)$ by minimizing $\left\|\ln z-\operatorname{Re}\left(\sum_{0}^{n} a_{k} z^{k}\right)\right\|_{\partial \Omega}$ w.r.t. the $a_{k}$ using the Lawson algorithm. The computations were carried out for different $n$, and the smallest error in the approximate conformal maps obtained was $3 \cdot 10^{-2}$. 
Example 4.2. Let $\partial \Omega=\{z=\cos (t)+i b \sin (t), 0 \leqslant t<2 \pi\}, b$ is a constant, $0<b<1$. A conformal mapping $\phi$ from $\Omega$ to the unit disk, $\phi(0)=0$, is given by an elliptic sine, see Kober [12, p. 177], which shows that the singularities of $\phi(z)$ closest to $\partial \Omega$ are poles at $\zeta_{1,2}= \pm i 2 b /\left(\sqrt{1-b^{2}}\right)$. As is shown in this and in the next example, it is sometimes possible to obtain good approximations of the singularities by locally approximating $\phi$ by a Möbius transformation $\phi_{M}$. In the present example, we consider the Möbius transformation that maps $0 \rightarrow 0$, and the circle of curvature through $i b$ onto the unit circle. It has a pole at

$$
\zeta_{1}^{*}:=i 2 b \frac{1-\frac{1}{2} b^{2}}{1-b^{2}}
$$

Note $\zeta_{1}^{*}=\zeta_{1}+O\left(b^{4}\right)$ as $b \rightarrow 0$. We approximate $\phi(z)\left(z-\zeta_{1}^{*}\right)\left(z-\bar{\zeta}_{1}^{*}\right)$ by polynomials. This reduces the error between a factor 5 and 10 in the following examples, compared to approximating $\phi(z)$ by polynomials.

$\begin{array}{cccc}b & n & \text { basis } & \left\|\left|\phi_{n}(z)\right|-1\right\|_{\partial \Omega} \\ 1 / 4 & 8 & \mathrm{M} & 1 \cdot 10^{-4} \\ 1 / 4 & 32 & \mathrm{~L} & 1 \cdot 10^{-6} \\ 1 / 8 & 16 & \mathrm{M} & 8 \cdot 10^{-5} \\ 1 / 8 & 32 & \mathrm{~L} & 5 \cdot 10^{-6}\end{array}$

When computations were done with a Lagrange basis, a monomial basis could not be used due to the ill-conditioning of the matrix (4.2).

The introduction of "approximate singularities" appears quite useful in the approximation of analytic function. In a different conformal mapping method, which also uses polynomials to approximate $\phi$, Levin et al. [13] reduced their approximation error by including extra basis functions with singularities at $\pm \zeta_{1}$ or at $\pm \zeta_{1}^{*}$.

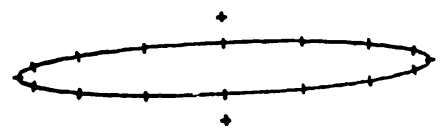

FIGURE 4.2

Figure 4.2 shows $\partial \Omega$ for $b=1 / 8$. The crosses mark $\pm \zeta_{1}^{*}$.

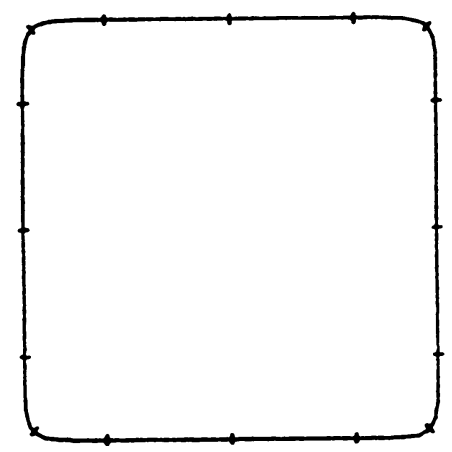

FIGURE 4.3 
Example 4.3. Let $\partial \Omega=\left\{z=x+i y, x^{4}+y^{4}=1\right\}$. Figure 4.3 shows $\partial \Omega$ and 16 Fejér points. $\left\|\left|\phi_{16}(z)\right|-1\right\|_{\partial \Omega}=6 \cdot 10^{-4}$. $\partial \Omega$ is circle-like enough to allow the power basis to be used even for $\phi_{32}$ and $\phi_{64}$.

Acknowledgment. This work is part of my thesis, and I wish to thank Germund Dahlquist for many valuable discussions.

Forschungsinstitut für Mathematik

ETH-Zentrum

CH-8092 Zürich, Switzerland

1. M. O. Afolabi \& K. O. Geddes, "Near-minimax approximation of analytic functions on regular polygons," Proc. 7th Manitoba Conf. on Numer. Math. and Comp., University of Manitoba, Winnipeg, 1977, pp. 163-176.

2. J. H. CURTISs, "Convergence of complex Lagrange interpolation polynomials on the locus of the interpolation points," Duke Math. J., v. 32, 1965, pp. 187-204.

3. S. W. EllacotT, "A technique for approximate conformal mapping," in Multivariable Approximation (D. C. Handscomb, ed.), Academic Press, London, 1978.

4. G. H. Elliotr, The Construction of Chebyshev Approximation in the Complex Plane, Thesis, University of London, 1979.

5. D. GAIER, “Integralgleichungen erster Art und konforme Abbildung," Math. Z., v. 147, 1976, pp. 113-129.

6. D. GAIER, Vorlesungen über Approximation im Komplexen, Birkhäuser, Basel, 1980.

7. W. GautschI, "The condition of orthogonal polynomials," Math. Comp., v. 26, 1977, pp. 923-924.

8. W. GAUTSCHI, "Questions of numerical condition related to polynomials," in Recent Advances in Numercial Analysis (C. de Boor and G. Golub, eds.), Academic Press, New York, 1978.

9. W. GautschI, "Condition of polynomials in power form," Math. Comp., v. 33, 1979, pp. 343-352.

10. K. O. GedDEs, "Chebyshev nodes for interpolation on a class of ellipses," in Theory of Approximation with Applications (A. G. Law and B. N. Sahney, eds.), Academic Press, New York, 1976.

11. K. O. Geddes \& J. C. MASON, "Polynomial approximation by projections on the unit circle," SIAM J. Numer. Anal., v. 12, 1975, pp. 111-120.

12. H. Kober, Dictionary of Conformal Representations, Dover, New York, 1957.

13. D. Levin, N. Papamichael \& A. Sideridis, "The Bergman kernel method for the numerical conformal mapping of simply connected domains," J. Inst. Math. Appl., v. 22, 1978, pp. 171-187.

14. J. C. MASON, "Recent advances in near-best approximation," in Approximation Theory III (E. W. Cheney, ed.), Academic Press, New York, 1980.

15. L. ReICHEL, "On the determination of boundary collocation points for some problems for the Laplace operator,” J. Comput. Appl. Math., v. 11, 1984, pp. 175-196.

16. L. Reichel, "A fast method for solving certain integral equations of the first kind with application to conformal mapping," J. Comput. Appl. Math. (To appear.) 